\title{
Temporary mechanical circulatory support for refractory heart failure: the German Heart Center Berlin experience
}

\author{
Gaik Nersesian $^{1}$, Felix Hennig ${ }^{1,2}$, Marcus Müller ${ }^{1}$, Johanna Mulzer ${ }^{1}$, Dmytro Tsyganenko ${ }^{1}$, \\ Christoph Starck ${ }^{1,2}$, Tom Gromann ${ }^{1}$, Volkmar Falk ${ }^{1,2,3}$, Evgenij Potapov ${ }^{1,2}$, Felix Schoenrath ${ }^{1,2}$ \\ ${ }^{1}$ Department of Cardiothoracic and Vascular Surgery, German Heart Center Berlin, Berlin, Germany; ${ }^{2}$ DZHK (German Centre for Cardiovascular \\ Research), Partner Site Berlin, Berlin, Germany; ${ }^{3}$ Department of Cardiothoracic Surgery, Charité-Universitätsmedizin Berlin, Corporate Member of \\ Freie Universität Berlin, Humboldt-Universität zu Berlin, and Berlin Institute of Health, Berlin, Germany \\ Correspondence to: Gaik Nersesian. Department of Cardiothoracic and Vascular Surgery, German Heart Center Berlin, Augustenburger Platz 1, 13353 \\ Berlin, Germany. Email: nersesian@dhzb.de.
}

\begin{abstract}
Background: Temporary mechanical circulatory support (MCS) offers a valuable option for treatment of refractory heart failure. We present our experience with selected MCS devices in cardiogenic shock of different etiologies.

Methods: We retrospectively studied patients who were treated in our institution between 01/2016 and 07/2018. Patients receiving only veno-arterial extracorporeal membrane oxygenation (VA-ECMO) support were excluded. Left ventricular support patients received Impella; right ventricular support was conducted using Levitronix CentriMag.

Results: Thirty-seven patients received an Impella left ventricular assist device (LVAD). Etiology was: acute on chronic ischemic cardiomyopathy (ICMP; $\mathrm{n}=12$ ), acute myocardial infarction (AMI; $\mathrm{n}=11$ ), dilated cardiomyopathy (DCMP; n=7) and toxic cardiomyopathy (TCMP; n=2). Two patients presented with postcardiotomy shock and acute myocarditis, respectively. In one case, Takotsubo cardiomyopathy was diagnosed. Impella was used solely in 28 patients (Impella group) with an in-hospital survival of $37 \%$. In nine patients, Impella was used in combination with extracorporeal life support (ECLS) implantation (ECMELLA group) - in-hospital survival was 33\%. In the Impella group six patients recovered, six received a long-term VAD and 16 died on device. In the ECMELLA group one patient recovered, three received a long-term VAD and five died. The majority of CentriMag implantations as a right ventricular assist device (RVAD) were necessary after LVAD implantation $(n=52)$; of these patients, 14 recovered, eight received long-term VAD and 30 died. The remaining 17 patients were supported by RVAD due to AMI (n=7); postcardiotomy $(n=7)$; right heart failure after heart transplantation $(n=2)$ and ICMP $(n=1)$. Six of these patients recovered, two required long-term VAD and nine died.

Conclusions: Survival after MCS implantation for left as well as right heart failure in cardiogenic shock remains low, but is superior to that of patients without mechanical support. Short-term MCS remains an option of choice if right, left or biventricular support is needed.
\end{abstract}

Keywords: Mechanical circulatory support (MCS); cardiogenic shock; Impella; CentriMag

Submitted Oct 02, 2018. Accepted for publication Nov 20, 2018.

doi: 10.21037/acs.2018.12.01

View this article at: http://dx.doi.org/10.21037/acs.2018.12.01

\section{Introduction}

Cardiogenic shock can present as isolated right, left or combined biventricular heart failure (HF), leading to lung edema and organ malperfusion, factors that have a direct impact on survival. In contemporary registries, decompensated dilated cardiomyopathy (DCMP) and ischemic cardiomyopathy (ICMP) together with acute myocardial infarction (AMI) are the main reasons for 


\begin{tabular}{|c|c|c|c|c|c|}
\hline Insertion & Percutaneous & $\begin{array}{l}\text { Percutaneous, } \\
\text { arterial access }\end{array}$ & Percutaneous & Sternotomy & $\begin{array}{l}\text { Percutaneous/ } \\
\text { septal puncture }\end{array}$ \\
\hline Max flow (L/min) & 7 & $5-5.5$ & No & 9.9 & $4.5-5$ \\
\hline Peripheral vascular injury & Low & Moderate & Very low & Low & Low \\
\hline Pump mechanism & Centrifugal & Axial & Pneumatic & Centrifugal & Centrifugal \\
\hline $\begin{array}{l}\text { Recommended } \\
\text { duration of use }\end{array}$ & 7 days & 10 days & Days-weeks & 30 days & 14 days \\
\hline
\end{tabular}

cardiogenic shock $(1,2)$. Currently available temporary mechanical circulatory support (MCS) devices are considered options for treatment of cardiogenic shock $(3,4)$ and provide a large variety of short-term support strategies; however, none of the contemporary devices has shown a significant outcome improvement and no randomized control trial of commercially available devices has be conducted so far (5).

Extracorporeal life support (ECLS) is the most common temporary MCS device, due to its reasonable cost and availability in every cardiothoracic surgery department as well as in many large cardiology departments with frequent acute percutaneous coronary intervention (PCI) procedures. Veno-arterial cannulation provides both oxygenation and circulation support. The peripheral placement, the option to install the support at the bedside and its suitability for use during cardiopulmonary bypass as an alternative to the heart-lung-machine makes ECLS an attractive option for circulatory support $(5,6)$.

A major disadvantage of ECLS is the lack of ventricular unloading. Furthermore, the outflow in the aorta creates an extremely high afterload, increasing the oxygen demand and wall tension of the myocardium, and thereby coronary perfusion worsens. Moreover, blood stasis in the left ventricle causes pulmonary hypertension, leading to interstitial lung edema $(5,7)$.

There are some options to improve ventricular unloading, starting with inotropic support and escalating to intra-aortic balloon pump (IABP) (8). However, this concept has failed to provide survival benefit in AMI patients in the
IABP Shock 2 trial $(9,10)$.

Therefore, a potential therapy of choice could be implantation of the Impella (AbioMed, Danvers, MA, USA) device $(5,7,11)$. Impella is a family of miniature rotatory pumps that can provide flow of 2.5 to $5 \mathrm{~L} / \mathrm{min}$, depending on the size of the vessel and the support needed. The Impella 5.0 pump, which is that most frequently used in our department, is placed through an arterial cut-down. With flow rates of up to $5 \mathrm{~L} / \mathrm{min}$ it can offer full support. Compared to ECLS, Impella fully unloads the left ventricle, preventing pulmonary edema and reducing the myocardial oxygen consumption. Moreover, Impella therapy requires much less anticoagulation than ECLS. The characteristics of modern left ventricular MCS devices are presented in Table 1 [adapted from (3-6)].

Most of the long-term implantable MCS devices were primarily designed for left ventricular support; nevertheless, proper right heart function plays a key role for the circulation. Up to $10-15 \%$ of patients develop acute right HF (RHF) after left ventricular assist device (LVAD) implantation, with subsequent need for biventricular support (12). A small group of patients may develop isolated RHF, mostly after acute myocardial infarction due to occlusion of the right coronary artery. Nowadays, four devices can be used for the therapy of RHF: ECLS, Tandem Heart (Cardiac Assist Inc., Pittsburgh, PA, USA), Impella RP (AbioMed) and CentriMag (Thoratec, Pleasanton, CA, USA) (5) (see Table 2) [adapted from (3-5)].

At the German Heart Center Berlin (Deutsches Herzzentrum Berlin, DHZB) the CentriMag centrifugal 


\begin{tabular}{llll}
\multicolumn{2}{l}{ Table 2 Short-term right ventricular support devices } & & TandemHeart \\
\hline Parameters & Impella RP & CentriMag & Double-lumen cannula \\
\hline Cannulation & $\begin{array}{l}\text { Single lumen cannula, right } \\
\text { atrium to pulmonary artery }\end{array}$ & $\begin{array}{l}\text { Two cannulas; central cannulation of the pulmonary } \\
\text { artery or percutaneous via r. jugular artery }\end{array}$ & Yes \\
Oxygenation & No & Yes & 4 \\
Max flow (L/min) & 4 & 9.9 & 4 \\
\hline
\end{tabular}

\begin{tabular}{lll}
\hline Table 3 Demographics & & \\
\hline Demographics & $\begin{array}{l}\text { Impella LVAD } \\
(\mathrm{n}=37)\end{array}$ & $\begin{array}{l}\text { CentriMag RVAD } \\
(\mathrm{n}=69)\end{array}$ \\
\hline Age, median [range] (years) & $59[18-76]$ & $57[20-77]$ \\
\hline Male, $\mathrm{n}$ [\%] & $24[65]$ & $48[70]$ \\
\hline Mean support, days & 10.5 & 21.5 \\
\hline Max. support, days & 69 & 118 \\
\hline $\begin{array}{l}\text { Previous heart-surgeries, } \mathrm{n} \\
\text { [\%] }\end{array}$ & $25[68]$ & $36[52]$ \\
\hline CPR, n [\%] & 9 [24] & $7[10]$ \\
\hline $\begin{array}{l}\text { LVAD, left ventricular assist device; RVAD, right ventricular assist } \\
\text { device; CPR, cardiopulmonary resuscitation. }\end{array}$
\end{tabular}

blood pump is considered the most advantageous MCS device for isolated right ventricular support. The magnetically levitated impeller minimizes the potential friction and shear forces on blood cells, preventing hemolysis. The maximal speed of 5,500 rpm allows blood flow of up to $9.9 \mathrm{~L} / \mathrm{min}$ to be generated. The impeller is built into a transparent plastic corpus, allowing visual evaluation of the flow. CentriMag provides a versatile mechanical support for right, left or biventricular HF; moreover, a membrane oxygenator can be added, providing pulmonary support (13). It is the most biocompatible system and is approved for up to four-week use (14), although it can be used successfully for a much longer time (15). CentriMag is recommended as a baseline biocompatibility control pump for future MCS devices (14).

Cannulation for the outflow cannula as a right ventricular assist device (RVAD) may be performed directly in the pulmonary artery, or through the wall of the right ventricle and pulmonary valve, or using a vascular graft attached to the pulmonary artery. Further, the long Bio-Medicus ${ }^{\circledR}$ femoral venous cannula (Medtronic Inc., Mounds View, MN, USA) may be placed percutaneously through the right internal jugular vein into the pulmonary artery under fluoroscopic or echocardiographic guidance $(6,16)$. The inflow cannula should drain the right atrium, mostly using peripheral venous access. Totally percutaneous access is also possible. See Table 2 for the access sites for the different devices.

Beside these pathophysiological considerations, concrete data of short term MCS devices in the setting of acute right and left HF remains scarce. The aim of this study is to further evaluate outcome parameters as well as potential advantages and disadvantages of short term MCS therapy.

\section{Methods}

We performed a single center retrospective analysis of a contemporary cohort of patients with cardiogenic shock, supported in our institution with Impella for short-term left ventricular circulatory support (01/2016 to $07 / 2018)$ and with CentriMag for short-term right ventricular support (01/2015 to $07 / 2018)$.

\section{Results}

\section{Impella LVAD}

The demographics of the patients are shown in Table 3.

In four $(11 \%)$ cases, left axillary artery access was used and in $28(76 \%)$ cases, the right axillary artery was accessed; in two $(5 \%)$ cases, the right femoral artery was punctured and in two cases, the left femoral artery was used.

In one $(3 \%)$ patient, due to high-grade stenosis of the axillary arteries and narrow femoral arteries, the Impella 5.0 device was inserted directly into the ascending aorta via a median sternotomy. In three cases, implantation was not successful due to a small axillary artery and in two, an Impella CP was inserted. In one case the Impella 2.5 was used.

Nine $(24 \%)$ patients were on ECLS during Impella implantation. In these cases, Impella was used as a vent. 


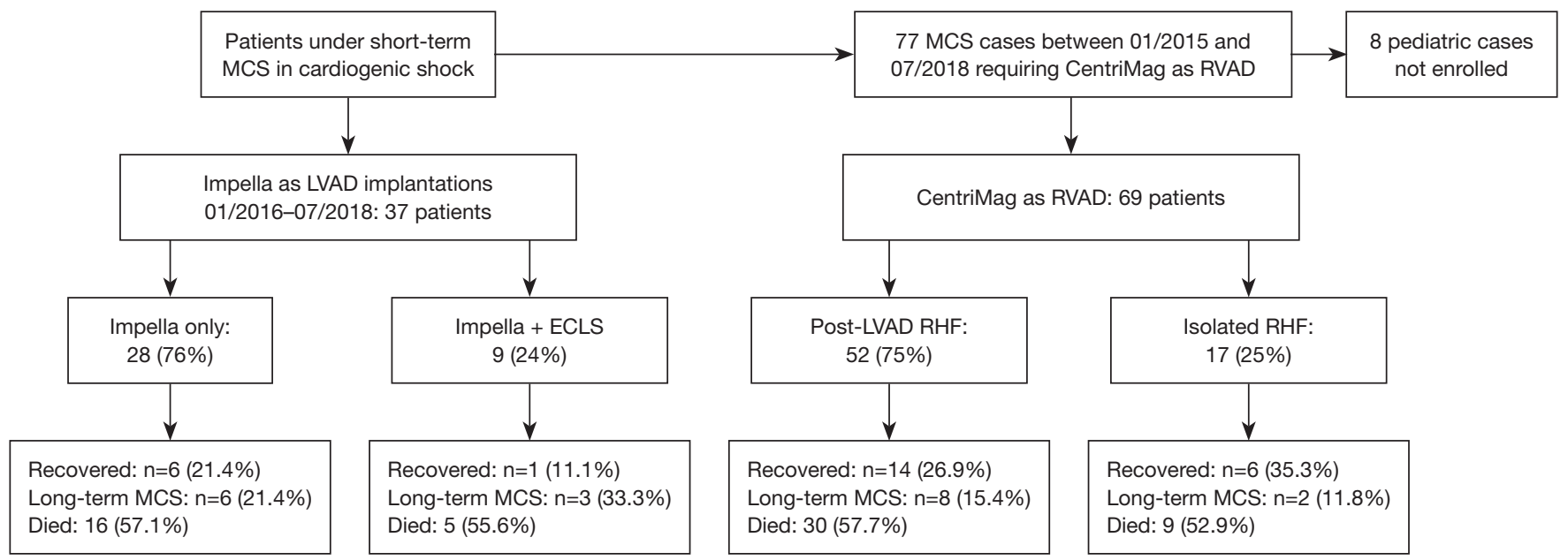

Figure 1 Flow diagram. Flow chart of the patient outcomes in different MCS groups. MCS, mechanical circulatory support; RVAD, right ventricular assist device; LVAD, left ventricular assist device; ECLS, extracorporeal life support; RHF, right heart failure.

\begin{tabular}{|lll}
\hline Table 4 Reason of death & \\
\hline Reason of death & Impella, $\mathrm{n}$ [\%] & CentriMag, $\mathrm{n}$ [\%] \\
\hline Total & $20[54]$ & $\mathrm{n}=39[57]$ \\
\hline Multi-organ failure & $9[45]$ & $24[62]$ \\
\hline Sepsis & $1[5]$ & $7[18]$ \\
\hline Cardiac arrest & $5[25]$ & $2[5]$ \\
\hline Cerebral bleeding & $3[15]$ & $4[10]$ \\
\hline Pulmonary failure & $1[5]$ & $2[5]$ \\
\hline RHF & $1[5]$ & 0 \\
\hline
\end{tabular}

$\mathrm{RHF}$, right heart failure.

Of these ECLS patients, three had ECLS explanted and Impella continued to work as an LVAD. In $28(76 \%)$ cases, Impella was used primarily as an LVAD. From the total 37 patients, nine were bridged to long-term VAD after mean support duration of 13.9 days (2-69 days), seven (19\%) were weaned after 16.6 days (5-25 days) and 20 died during support (mean 6.54 days; range, 19 h to 43 days). Nine (24\%) patients received an IABP implantation before Impella; in eight $(22 \%)$ cases severe hemolysis prompted a pump explantation. The patient outcomes are presented in Figure 1.

The overall mortality on device was $54 \%$ (Table 4).

\section{CentriMag RVAD}

The demographics of the CentriMag group are shown in

\begin{tabular}{|lll|}
\hline Table 5 Etiology of cardiogenic shock & \\
\hline Etiology & $\begin{array}{l}\text { Predominantly left } \\
\text { heart failure }(\mathrm{n}=37), \mathrm{n}(\%)\end{array}$ & $\begin{array}{l}\text { CentriMag } \\
(\mathrm{n}=69), \mathrm{n}(\%)\end{array}$ \\
\hline DCMP & $7(19.0)$ & $23(33.3)$ \\
\hline ICMP & $12(32.4)$ & $16(23.2)$ \\
\hline AMI & $11(29.7)$ & $14(20.3)$ \\
\hline TCMP & $2(5.4)$ & $1(1.4)$ \\
\hline Postcardiotomy & $2(5.4)$ & $10(14.5)$ \\
\hline Acute myocarditis & $2(5.4)$ & $1(1.4)$ \\
\hline Takotsubo disease & $1(2.7)$ & 0 \\
\hline $\begin{array}{l}\text { Congenital heart } \\
\text { disease }\end{array}$ & 0 & $2(2.9)$ \\
\hline $\begin{array}{l}\text { Post-transplantation } \\
\text { heart failure }\end{array}$ & 0 & \\
\hline
\end{tabular}

DCMP, dilated cardiomyopathy; ICMP, ischemic cardiomyopathy; AMI, acute myocardial infarction; TCMP, toxic cardiomyopathy.

Table 5 and the patients' outcomes are presented in Figure 1.

In $62(90 \%)$ cases, direct access to the pulmonary artery (39 cases through vascular graft) was chosen for blood return and in seven $(10 \%)$, percutaneous cannulation through the jugular vein $(6,16)$. In $48(70 \%)$ cases, peripheral cannulation for venous drainage was employed and in 21 , the RA was directly cannulated.

Of 69 patients, ten $(14.5 \%)$ were transited to long-term RVAD after a mean of 61.9 [18-118] days, 20 (29\%) were 


\begin{tabular}{lcl}
\multicolumn{3}{l}{ Table 6 Shock parameters related to Impella implantation } \\
\hline Parameters & $\mathrm{N}$ & Survival, $\mathrm{n}[\%]$ \\
\hline CPR & 9 & $2[22]$ \\
Blood $\mathrm{pH}<7.2$ & 3 & $0[0]$ \\
Blood $\mathrm{pH}>7.45$ & 5 & $1[20]$ \\
\hline CPR, cardiopulmonary resuscitation. &
\end{tabular}

\begin{tabular}{lll}
\multicolumn{2}{l}{ Table 7 Complications during support } \\
\hline Complications & $\begin{array}{l}\text { Impella } \\
(\mathrm{n}=37), \mathrm{n}[\%]\end{array}$ & $\begin{array}{l}\text { CentriMag } \\
(\mathrm{n}=69), \mathrm{n}[\%]\end{array}$ \\
\hline Major bleeding & $4[11]$ & $24[35]$ \\
\hline Hemolysis/pump thrombosis & $8[22]$ & $8[12]$ \\
Stroke & $5[14]$ & $4[6]$ \\
\hline Infections & $4[11]$ & $28[41]$ \\
\hline Peripheral ischemia & $2[5]$ & 0 \\
\hline
\end{tabular}

weaned after 22.6 [5-76] days and 39 died after a mean of 10.6 [0.5-51] days of support. A 30-day survival of $45 \%$ was demonstrated.

Thirty-nine (57\%) patients died on CentriMag support (Table 4).

\section{Discussion}

Despite the latest improvements in short-term MCS devices and the rising number of implantations, the therapy of acute refractory HF remains challenging, with high mortality rates.

The etiology of HF plays a key role for the MCS therapy choice. In our study we describe two different patient groups with left and with right ventricular failure, leading to cardiogenic shock. In patients suffering from cardiogenic shock caused predominantly by left ventricular failure, the Impella LVAD is a valuable option as a bridge to decision or recovery. However, "crash and burn" patients (Intermacs profile 1) and patients under cardiopulmonary resuscitation (CPR) or with evident multi-organ failure, especially with lung failure, should be treated by ECLS. Full cardiopulmonary support and rapid installation play a crucial role in these patients (17). Between 01/2012 and $01 / 2018$ in our institution 714 patients were supported with ECLS. Patients who underwent long-term LVAD implantation after hemodynamic stabilization on ECLS present $62 \% 30$-day survival and $43 \%$ one-year survival, which is acceptable in the setting of severe cardiogenic shock (18).

The successful use of Impella devices during high-risk PCI and reduced mortality rates in at least smaller trials made it an attractive therapy strategy in cardiology $(19,20)$. However, its preventive use completely differs from the therapy of patients in manifest cardiogenic shock. The majority of patients in previous studies developed cardiogenic shock after AMI or postcardiotomy. Survival in this setting was around $50 \%(11,21)$. Early implantation in cardiogenic shock is crucial and has to be aimed for (22); on the other hand, futility has to be avoided. Therefore, timely referral and adequate evaluation are cornerstone. Comparison of survival rates is difficult, since etiology and exclusion criteria as prescribed above differ, but compared to previous studies, survival tends to be higher if patients are supported with the Impella device $(23,24)$. Some factors such as resuscitation before or during Impella therapy or pathological venous blood $\mathrm{pH}$ on admission may be considered as low survival predictors (Table 6). In cases of postcardiotomy cardiogenic shock, the threshold for the decision should be moved toward earlier implantation to avoid multi-organ failure (17). However, there is no evidence that a more aggressive approach would improve the outcome. Adverse events under support occurred in $43 \%$ of patients with Impella support and are therefore comparable to the rate with ECLS therapy (24) (Table 7).

Nevertheless, second-line Impella 5.0 implantation can be successfully performed in patients showing no myocardial recovery on prolonged ECLS support or suffering from complications of ECLS therapy (25).

A great potential of Impella use is the step-up approach in patients already supported by ECLS. This approach was used in our cohort. In nine patients who underwent Impella implantation in the setting of $\mathrm{LV}$ distention only one case of bleeding, infection and stroke (respectively) was diagnosed (this complications are associated with Impella therapy only). A multicenter study by Pappalardo et al. demonstrated a significant improvement of outcomes in patients who underwent simultaneous ECLS and Impella (so called ECMELLA) therapy, compared with VA-ECLS use only $(26,27)$. The recent retrospective single center study by Patel et al. also showed a reduction in mortality rates in ECMELLA patients. Decreasing use of inotropic support and a lower complication rate were also reported (6).

In June 2018, Pappalardo et al. reported biventricular circulatory support with Impella CP and Impella RP in a patient 
with acute cardiogenic shock, which developed after ischemic stroke, complicated with pulmonary hypertension (28). The simultaneous use of two Impella devices in a setting of biventricular support could be a promising alternative compared to more invasive methods; however, the difficulties in placement and the lack of experience with Impella RP, especially in a prolonged therapy concept, remain a limiting factor. Therefore, we consider CentriMag right heart support as the current option of choice. Among our patients, maximal right ventricular support with CentriMag of 118 days was demonstrated. The longest CentriMag therapy of 304 days was described by Griffith et al. in 2012 (15).

The mortality of patients supported with CentriMag RVAD is expected to be high, since many patients who develop RHF after LVAD implantation have previously had chronic RV failure, are predominantly in Intermacs profile 1 or 2 or have been previously supported with ECLS. In a meta-analysis by Borisenko et al., 30-day survival of $61 \%$ (46-75\%) was demonstrated for this group of patients (29). Simultaneous use of long-term LVAD with short-term RVAD in the setting of biventricular support might be considered to be more advantageous than primary biventricular assist device (BiVAD) implantation because of the ability to wean a relevant proportion of patients after right heart recovery and therefore potentially fewer long-term complications. Nowadays there is no continuous flow longterm ventricular assist device (cfVAD) designed for right ventricular support available. Therefore, LVADs such as HeartMate 3 and HeartWare have to be implanted (30). In a work by Eulert Grehn from our center, patients who underwent primary cfVAD implantation were compared with those who received subsequent implantation after short-term right ventricular support, including with the CentriMag pump. The primary cfVAD implantation has shown no significant increase in survival compared with the subsequent implantation: $72.7 \%$ vs. $71.4 \%$ in 30-day survival (31). Prospective and randomized clinical trials comparing the outcomes of primary and subsequent cfVAD implantation in the setting of biventricular heart support are needed; however, short-term RVAD therapy is a valuable option for a selected group of patients in postLVAD RHF. In this setting CentriMag can be used as an approved therapy.

\section{Limitations}

The present article reports a single-center experience in a small cohort of consecutive patients; results should be confirmed in larger multicenter randomized studies.

\section{Conclusions}

Impella for left ventricular support employed in cardiac surgery departments is associated with elevated mortality compared to preventive use during high risk PCI. However, the vast majority of our patients are in profound cardiogenic shock prior to the surgery and, in this setting, the Impella 5.0 implantation can provide sufficient circulatory support with acceptable complication rates.

The CentriMag as a temporary RVAD in the setting of post-LVAD right ventricular failure provides a valuable option if prolonged support is needed and stimulates right heart recovery, which might allow patients to be weaned from the device.

Future studies should evaluate criteria for RV recovery on temporary RVAD. A prospective randomized study comparing different types of temporary MCS in cardiogenic shock would be helpful in improving our treatment of these high-risk patients.

\section{Acknowledgements}

The authors thank Anne Wölffel-Gale for editorial assistance and Christine Detschades for data collection.

\section{Footnote}

Conflicts of Interest: The authors have no conflicts of interest to declare.

\section{References}

1. Kirklin JK, Pagani FD, Kormos RL, et al. Eighth annual INTERMACS report: Special focus on framing the impact of adverse events J Heart Lung Transplant 2017;36:1080-6.

2. Kirklin JK, Xie R, Cowger J, et al. Second annual report from the ISHLT Mechanically Assisted Circulatory Support Registry. J Heart Lung Transplant 2018;37:685-91.

3. Ando M, Garan AR, Takayama H. et al. A continuous flow external ventricular assist device for cardiogenic shock: Evolution over 10 years. J Thorac Cardiovasc Surg 2018;156:157-65.e1.

4. Csepe TA, Kilic A. Advancements in mechanical 
circulatory support for patients in acute and chronic heart failure. J Thorac Dis 2017;9:4070-83.

5. Shishehbor MH, Moazami N, Tong MZ, et al. Cardiogenic shock: From ECMO to Impella and beyond. Cleve Clin J Med 2017;84:287-95.

6. Patel SM, Lipinski J, Al-Kindi SG, et al. Simultaneous Venoarterial Extracorporeal Membrane Oxygenation and Percutaneous Left Ventricular Decompression Therapy with Impella Is Associated with Improved Outcomes in Refractory Cardiogenic Shock. ASAIO J 2019;65:21-8.

7. Ma P, Zhang Z, Song T, et al. Combining ECMO with IABP for the treatment of critically Ill adult heart failure patients. Heart Lung Circ 2014;23:363-8.

8. Keebler ME, Haddad EV, Choi CW, et al. Venoarterial Extracorporeal Membrane Oxygenation in Cardiogenic Shock. JACC Heart Fail 2018;6:503-16.

9. Pappalardo F, Ajello S, Greco M, et al Contemporary applications of intra-aortic balloon counterpulsation for cardiogenic shock: a "real world" experience. J Thorac Dis 2018;10:2125-34.

10. Thiele H, Schuler G, Neumann FJ, et al. Intraaortic balloon counterpulsation in acute myocardial infarction complicated by cardiogenic shock: Design and rationale of the Intraaortic Balloon Pump in Cardiogenic Shock II (IABP-SHOCK II) trial. Am Heart J 2015;169:e7-8.

11. Gaudard P, Mourad M, Eliet J, et al. Management and outcome of patients supported with Impella 5.0 for refractory cardiogenic shock. Crit Care 2015;19:363.

12. Dandel M, Krabatsch T, Falk V. Left ventricular vs. biventricular mechanical support: Decision making and strategies for avoidance of right heart failure after left ventricular assist device implantation. Int J Cardiol 2015;198:241-50.

13. Takeda K, Garan AR, Ando M, et al. Minimally invasive CentriMag ventricular assist device support integrated with extracorporeal membrane oxygenation in cardiogenic shock patients: a comparison with conventional CentriMag biventricular support configuration. Eur J Cardiothorac Surg 2017;52:1055-61.

14. Chan CH, Pieper IL, Hambly R, et al. The CentriMag centrifugal blood pump as a benchmark for in vitro testing of hemocompatibility in implantable ventricular assist devices. Artif Organs 2015;39:93-101.

15. Griffith KE, Jenkins E, Stulak J, et al. Long-term use of the CentriMag® Ventricular Assist System as a right ventricular assist device: a case report. Perfusion 2012;27:65-70.

16. Kazui T, Tran PL, Echeverria A, et al. Minimally invasive approach for percutaneous CentriMag right ventricular assist device support using a single PROTEKDuo Cannula. J Cardiothorac Surg 2016;11:123.

17. Hennig F, Potapov EV, Falk V, et al. Rapid Application Is Crucial. Dtsch Arztebl Int 2016;113:39-40.

18. Tsyganenko D, Gromann TW, Schoenrath F, et al. Predictors of mid-term outcomes in patients undergoing implantation of a ventricular assist device directly after extracorporeal life support. Eur J Cardiothorac Surg 2018. [Epub ahead of print].

19. Russo G, Burzotta F, D'Amario D, et al. Hemodynamics and its predictors during Impella-protected PCI in high risk patients with reduced ejection fraction. Int J Cardiol 2019;274:221-5.

20. Kovacic JC, Kini A, Banerjee S, et al Patients with 3-vessel coronary artery disease and impaired ventricular function undergoing PCI with Impella 2.5 hemodynamic support have improved 90-day outcomes compared to intra-aortic balloon pump: a sub-study of the PROTECT II trial. J Interv Cardiol 2015;28:32-40

21. Takayama H, Soni L, Kalesan B, et al. Bridge-to-decision therapy with a continuous-flow external ventricular assist device in refractory cardiogenic shock of various causes. Circ Heart Fail 2014;7:799-806.

22. Lund LH. The continued value of risk scores in advanced heart failure. Kardiol Pol 2018;76:1299-300.

23. Ouweneel DM, Eriksen E, Sjauw KD, et al. Percutaneous Mechanical Circulatory Support Versus Intra-Aortic Balloon Pump in Cardiogenic Shock After Acute Myocardial Infarction. J Am Coll Cardiol 2017;69:278-87.

24. Ouweneel DM, Schotborgh JV, Limpens J, et al. Extracorporeal life support during cardiac arrest and cardiogenic shock: a systematic review and meta-analysis. Intensive Care Med 2016;42:1922-34

25. Schibilsky D, Kruger T, Lausberg HF, et al. Impella 5.0 as a Second-Line Mechanical Circulatory Support Strategy After Extracorporeal Life Support. Artif Organs 2016;40:909-16

26. Pappalardo F, Schulte C, Pieri M, et al. Concomitant implantation of Impella ${ }^{\circledR}$ on top of veno-arterial extracorporeal membrane oxygenation may improve survival of patients with cardiogenic shock. Eur J Heart Fail 2017;19:404-12.

27. Tschöpe C, Van Linthout S, Klein O, et al. Mechanical Unloading by Fulminant Myocarditis: LV-IMPELLA, ECMELLA, BI-PELLA, and PROPELLA Concepts. J Cardiovasc Transl Res 2018. [Epub ahead of print].

28. Pappalardo F, Scandroglio AM, Latib A. Full percutaneous 
biventricular support with two Impella pumps: the Bi-Pella approach. ESC Heart Fail 2018;5:368-71.

29. Borisenko O, Wylie G, Payne J, et al. Thoratec CentriMag for temporary treatment of refractory cardiogenic shock or severe cardiopulmonary insufficiency: a systematic literature review and meta-analysis of observational studies. ASAIO J 2014;60:487-97

30. Lavee J, Mulzer J, Krabatsch T, et al. An international

Cite this article as: Nersesian G, Hennig F, Müller M, Mulzer J, Tsyganenko D, Starck C, Gromann T, Falk V, Potapov E, Schoenrath F. Temporary mechanical circulatory support for refractory heart failure: the German Heart Center Berlin experience. Ann Cardiothorac Surg 2019;8(1):76-83. doi: 10.21037/acs.2018.12.01 multicenter experience of biventricular support with HeartMate 3 ventricular assist systems. J Heart Lung Transplant 2018;37:1399-402.

31. Eulert-Grehn JJ, Lanmüller P, Schönrath F, et al. Two implantable continuous-flow ventricular assist devices in a biventricular configuration: technique and results. Interact Cardiovasc Thorac Surg 2018;27:938-42. 